\title{
Análise de falhas de um gerador de vapor usando as ferramentas FMEA e FTA
}

\author{
Analysis of a steam generator using the \\ FMEA and FTA techniques
}

Beatriz Ottoni Azevedo Porto Miranda ${ }^{1}$, Antonio Carlos Barbosa Zancanella ${ }^{1}$, Rômulo Maziero ${ }^{2}$, Bruno Dorneles de Castro ${ }^{2}$, Juan Carlos Campos Rubio ${ }^{2}$

\footnotetext{
${ }^{1}$ Instituto Federal de Educação, Ciência e Tecnologia do Espírito Santo, Rodovia BR Norte Km 58, 101, Litorâneo, CEP: 29932-540, São Mateus, ES, Brasil.

${ }^{2}$ Programa de Pós-graduação em Engenharia Mecânica, Universidade Federal de Minas Gerais, Av. Pres. Antônio Carlos, 6627, Pampulha, CEP: 31270-901, Belo Horizonte, MG, Brasil.

e-mail: beatrizoapmiranda@gmail.com, antonio.zancanella@ifes.edu.br, maziero@ufmg.br, brunodorn_92@hotmail.com,juan@ufmg.br
}

\section{RESUMO}

A integridade estrutural de um gerador de vapor pode ser afetada por diversos fatores, como o tipo de material que compõe a estrutura, a qualidade das juntas soldadas, os parâmetros de fabricação da caldeira e os procedimentos operacionais.Neste contexto, a corrosão se destaca como uma das principais fontes de degradação podendo levar essas superfícies sob pressão a um completo colapso. Neste estudo, foi realizada uma análise de falhas para investigar o processo que leva à ruptura e à corrosão em uma caldeira, utilizando as ferramentas FMEA (Análise de modos de falhas e efeitos) e FTA (Análise da árvore de falhas). Ensaios metalográficos de amostras da estrutura e um estudo físico-químico da água de alimentação do sistema também foram analisados. Os ensaios metalográficos evidenciaram pequenas fissuras no metal de base, além de falta de penetração na junta soldada e um desalinhamento de junta. A análise da água mostrou uma alta concentração de íons cloreto e um baixo pH da água de alimentação, fatores que causam a ruptura da camada passiva e resultam em corrosão. O uso das ferramentas FMEA e FTA possibilitaram a compreensão de fatores que atuam como concentradores de tensão, associados à inferior qualidade da água de alimentação do gerador de vapor e erros durante o processo de soldagem, que resultaram em corrosão localizada seguida de ruptura e vazamento de água líquida e em vapor.

Palavras-chave: Caldeiras, corrosão, FMEA, FTA.

\begin{abstract}
The structural health of a steam generator can be affected by several factors, such as the type of material of the structure, the quality of weld joints made, the boiler manufacturing parameters and the operating procedures performed. In this context, corrosion stands out as one of the major sources of degradation and can lead a surface under pressure to complete collapse. In this study, a failure analysis was made to investigate the process that lead to rupture and corrosion in a steam generator, using FMEA (Failure Mode and Effects Analysis) and FTA (Fault Tree Analysis) techniques. Metallographic tests of structure and a physicochemical study of the system feed water were also analysed. The metallographic tests showed small cracks in the base metal, in addition to lack of penetration in the welded joint and a misalignment of the joint. Water analysis showed a high concentration of chloride ions and a low $\mathrm{pH}$ of the feedwater, factors that cause the rupture of the passive layer and result in corrosion. The FMEA and FTA techniques allowed the understanding of the factors that act as stress concentrators, associated with lower quality of the steam generator feed water and errors during the welding process, which resulted in localized corrosion followed by rupture and leakage of liquid and steam water.
\end{abstract}

Keywords: Boilers, corrosion, FMEA, FTA. 


\section{INTRODUÇÃO}

O uso de caldeiras em processos de esterilização é largamente difundido, devido ao ótimo custo-benefício na esterilização de artigos termossensíveis. Composto por uma câmara de aço inoxidável, o equipamento conta com uma ou duas portas, além de uma válvula de segurança, um manômetro e um indicador de temperatura. A temperatura da caldeira é equivalente ao ponto de ebulição da água (de 121 a $\left.135{ }^{\circ} \mathrm{C}\right)$. O tempo, a temperatura e a pressão de trabalho são variáveis manipuláveis, que podem levar ao alcance de temperaturas adequadas para esterilização [1].

Para o correto funcionamento das caldeiras, essas máquinas devem apresentar boas propriedades de resistência à corrosão, à fluência e à fadiga. Em um processo de soldagem com qualidade, se aumenta a confiabilidade desses equipamentos durante a operação. As juntas soldadas apresentam um processo de desgaste diferente em comparação ao metal base, e por isso merecem uma atenção especial em relação ao processo de degradação por corrosão [2]. Dessa forma, o uso de aços inoxidáveis austeníticos são amplamente aplicados nessas estruturas [3, 4]. Uma das características mais relevantes dos aços inoxidáveis AISI 316L e 304, por exemplo, é a excelente resistência à corrosão quando comparados aos aços convencionais. Entretanto as ligas do tipo $\mathrm{Fe}-\mathrm{Cr}-\mathrm{Ni}$ não são imunes a esse tipo de dano, sendo suscetíveis principalmente à corrosão localizada [5,6]. Uma das principais características quanto à superfície dos aços inoxidáveis é que estes formam naturalmente uma camada passiva aderente e impermeável quando expostos ao ar, a qual é responsável pela resistência à corrosão destas ligas, já que representa uma barreira física entre a superfície metálica e o ambiente [5] .

Considerando o processo de operação da máquina, o uso de ferramentas como análise de modos de falhas e efeitos (FMEA) e análise da árvore de falhas (FTA) pode contribuir para uma maior confiabilidade do processo. As mesmas possibilitam um conhecimento mais aprofundado sobre a função de cada componente analisado, os possíveis defeitos e a relação entre causa e efeito. Essas técnicas possibilitam uma tomada de decisão mais precisa em termos de custo e de qualidade no desempenho [7, 8].

Neste contexto, o objetivo deste trabalho foi realizar uma análise de falhas para investigar a corrosão e a ruptura de um gerador de vapor, utilizando as técnicas de FMEA e FTA. Foram realizadas também análises metalográficas de amostras do metal base e da junta soldada, e uma análise físico-química da água de alimentação, com o intuito de compreender os fenômenos que resultam em falhas na estrutura da caldeira.

\section{MATERIAIS E MÉTODOS}

O material investigado neste estudo foi uma chapa de aço inoxidável austenítico da série 304 (espessura de $4,17 \pm 0,012 \mathrm{~mm}$ na região lateral, $3,15 \pm 0,044 \mathrm{~mm}$ na região frontal e $3,16 \pm 0,028 \mathrm{~mm}$ na região posterior) utilizada na fabricação do gerador de vapor, usado em um sistema de esterilização composto por três resistores elétricos, com uma potência de $12 \mathrm{~kW}$, atuando na pressão de trabalho de $2,5 \mathrm{kgf} \mathrm{cm}^{-2}$ e na faixa de temperatura de 121 a $134^{\circ} \mathrm{C}$, conforme estudado por MIRANDA et al. [9].

As imagens internas da caldeira foram obtidas usando um boroscópio industrial ECO-610 Ecogage e uma sonda de $5,50 \mathrm{~mm}$. As imagens foram investigadas com objetivo de avaliar a qualidade superficial da parte da estrutura que entra em contato direto com a água de alimentação, contribuindo para a compreensão de fenômenos corrosivos presentes no ambiente interno da caldeira.

Três amostras de seção transversal de uma junta de solda lateral do gerador de vapor foram removidas para análises de macrografia e micrografia (Figura 1a). As amostras foram lixadas (uso sequencial de lixas $220,320,400,600,800$ e 1200) e polidas com alumina de 1,0 e $0,3 \mu \mathrm{m}$. Posteriormente, as amostras foram submetidas a um ataque eletrolítico (Figura 1b), usando uma fonte ajustada de $5 \mathrm{~V}$ e uma solução de $10 \% \mathrm{em}$ ácido oxálico. Para análise da microestrutura, com ampliações de 100 e 200x, foi utilizado um microscópio Olympus BX51M (Figura 1c).

Para caracterizar a água de alimentação utilizada no gerador de vapor, foi realizada uma análise físicoquímica da água. O procedimento foi realizado pela empresa FULLIN - Laboratório de Análise Agronômica, Ambiental e Preparo de Soluções Químicas (Espírito Santo, Brasil).

As ferramentas FMEA e FTA foram utilizadas para detalhar o processo de ruptura (modo de falha) da estrutura da máquina, de modo a possibilitar o levantamento de ações que possam retardar ou eliminar as causas desse problema. As análises possibilitam uma maior confiabilidade do processo, uma vez que evidencia as relações entre causas e efeitos de falhas em estruturas, contribuindo para um maior conhecimento do processo em estudo. Além disso, elas permitem uma melhor tomada de decisão, em termos de custo e desempenho, de uma forma simples e aprofundada, uma vez que facilitam o desdobramento de causas e efeitos de uma modo de falha e melhoram a eficiência das medidas gerenciais para evitar uma nova ocorrência dessa falha. 


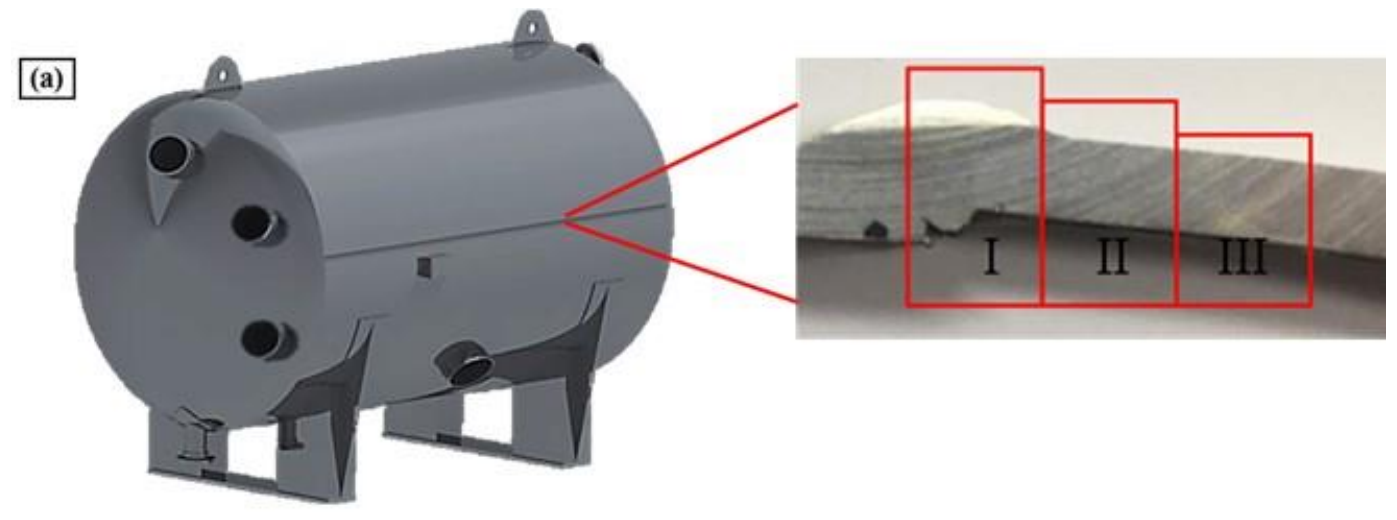

(b)
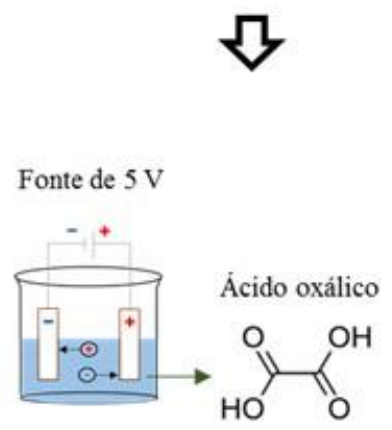

(c)

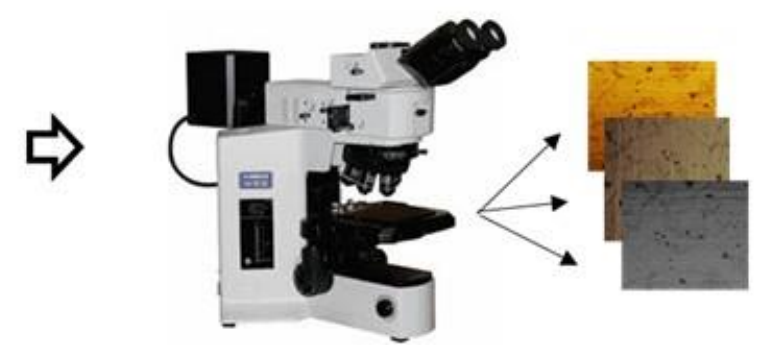

Figura 1: Processo de análise por micrografias: (a) coleta de amostras das regiões I, II e III, (b) ataque eletrolítico e (c) uso de microscópio para obtenção das micrografias (Adaptado de Miranda et al. [9]).

\section{RESULTADOS E DISCUSSÕES}

\subsection{Análise superficial interna}

A análise da superfície interna da caldeira (Figura 2) possibilitou a visualização de forte incrustação nas regiões de solda com penetração excessiva (Figura 2a), falta de penetração em outras áreas da superfície (Figura 2b), além de corrosão localizada (Figura 2c), conforme WANG et al. [10]. Os defeitos verificados ressaltam a necessidade de uma manutenção corretiva na estrutura da máquina, uma vez que podem concentrar tensões e levar à falha prematura da estrutura. 


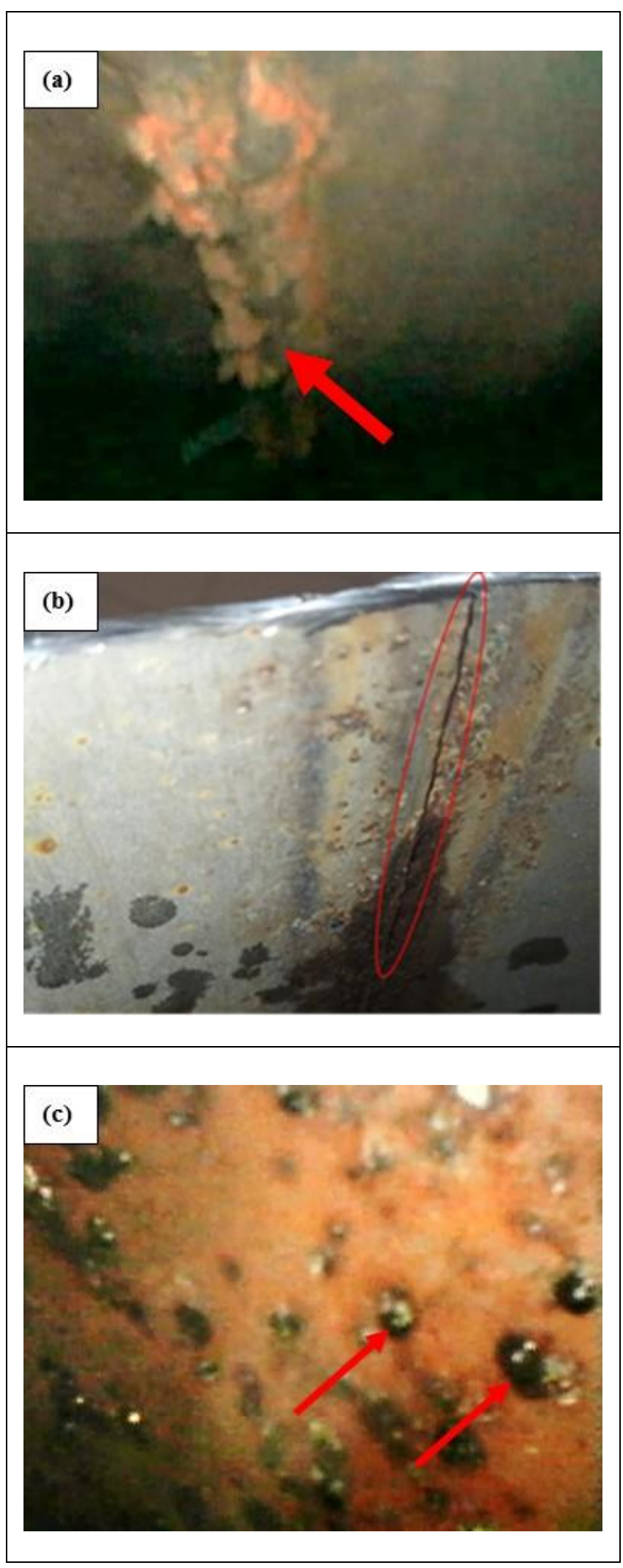

Figura 2: (a) Cordão com excesso de material de adição e presença de incrustações, (b) falta de material de adição e (c) corrosão por pites.

\subsection{Análise metalográfica}

A macrografia das regiões analisadas revelou a situação da integridade superficial da zona termicamente afetada (ZTA) e do metal base. Com base nessa análise, foi possível visualizar a presença de pequenas 
fissuras no metal de base, falta de penetração do material fundido e um desalinhamento de junta (Figura 3). Essas descontinuidades denotam a presença de concentradores de tensão e podem favorecer o início de uma fratura frágil na estrutura.

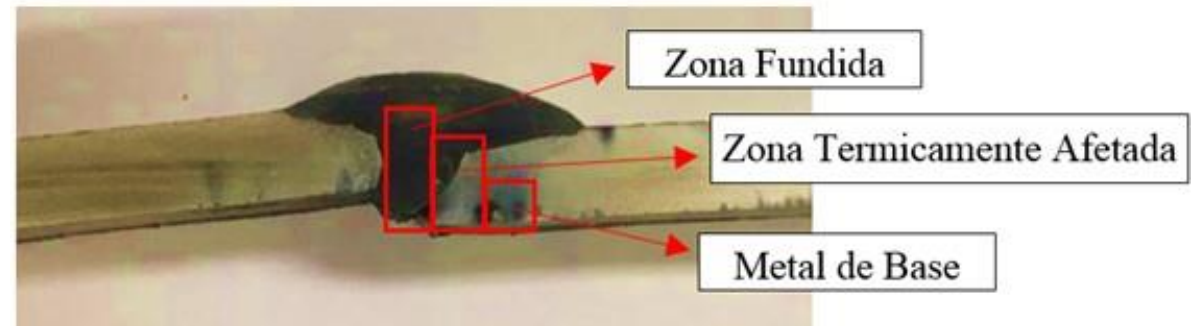

Figura 3: Junta soldada após ataque químico.

As micrografias foram realizadas em três regiões básicas de uma amostra submetida ao processo de soldagem a arco elétrico com eletrodo revestido (Figura 4). . Primeiramente, salienta-se a existência de uma zona fundida $(\mathrm{ZF})$ e mais escura, região na qual ocorreu o fusão e a solidificação durante a operação, apresentando uma estrutura primária de grãos colunares. A ZTA (Região I), por sua vez, mostra uma alteração na microestrutura do metal base, em função do ciclo térmico de soldagem, caracterizado pelo crescimento dos grãos na fronteira entre ZF e ZTA (Região II) [11 - 13]. Por fim, a região do metal base (Região III) não se mostrou afetada pelo processo de soldagem.
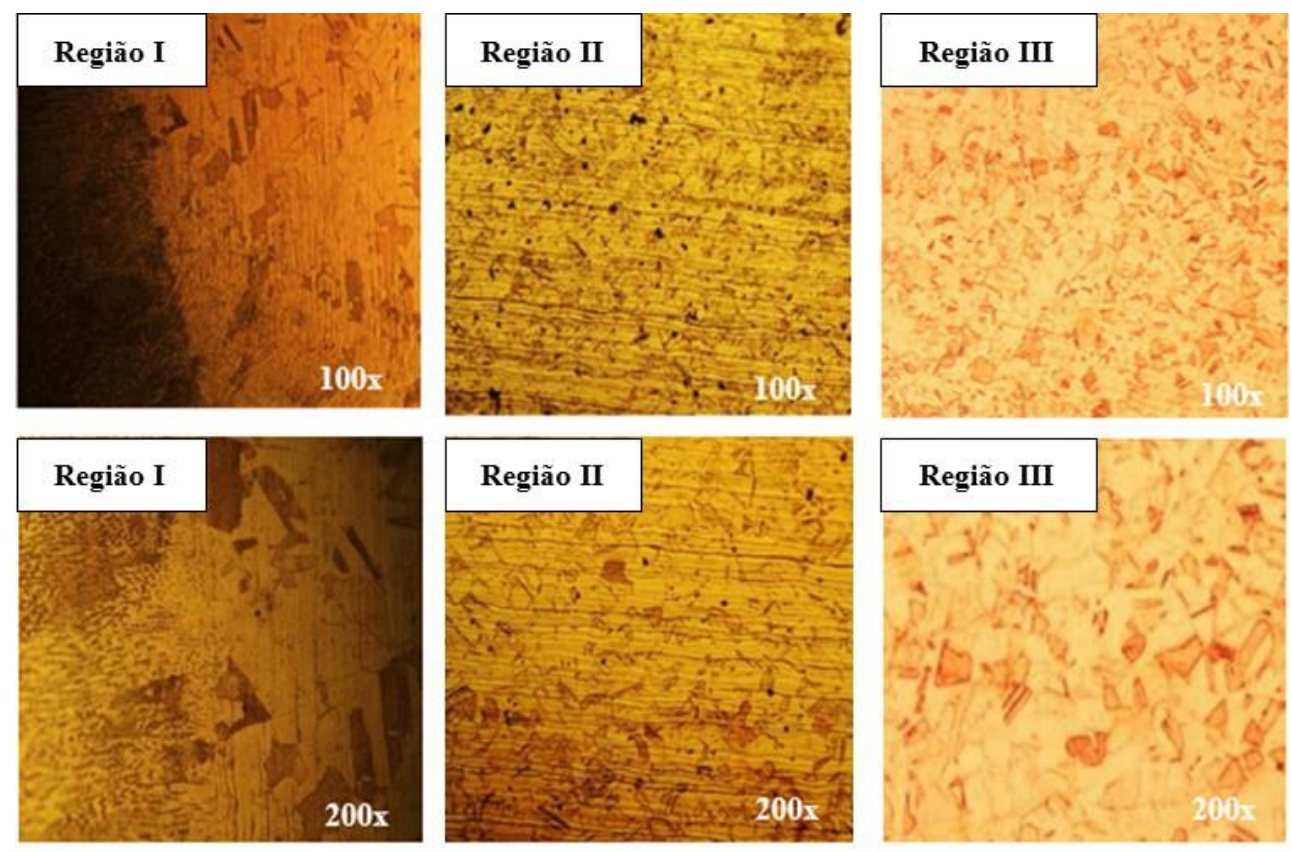

Figura 4: Micrografias das regiões amostrais I, II e III.

\subsection{Análise físico-química da água de alimentação}

A Tabela 1 apresenta os valores dos parâmetros obtidos a partir da análise físico-química da água utilizada pela caldeira.

Tabela 1: Análise físico-química da água de alimentação do gerador de vapor.

\begin{tabular}{l|l}
\hline PARÂMETROS & VALORES \\
\hline Alumínio total & $0,826 \mathrm{mg} \mathrm{L}^{-1}$ \\
\hline Cloreto & $36,9 \mathrm{mg} \mathrm{L}^{-1}$ \\
\hline Cloro residual livre & $0,6 \mathrm{mg} \mathrm{L}^{-1}$ \\
\hline
\end{tabular}




\begin{tabular}{l|l}
\hline Cor aparente & $79 \mathrm{mg} \mathrm{Pt}^{-C o ~ L}$ \\
\hline Dureza total (calc.) & $24,75 \mathrm{mg} \mathrm{L}^{-1}$ \\
\hline Ferro total & $2,05 \mathrm{mg} \mathrm{L}^{-1}$ \\
\hline Fluoreto & $0,6 \mathrm{mg} \mathrm{L}^{-1}$ \\
\hline Manganês total & $0,66 \mathrm{mg} \mathrm{L}^{-1}$ \\
\hline Nitrogênio amoniacal & $<0,150 \mathrm{mg} \mathrm{L}^{-1}$ \\
\hline pH & 6,18 \\
\hline Sódio total & $19 \mathrm{mg} \mathrm{L}^{-1}$ \\
\hline Sólidos dissolvidos totais & $78 \mathrm{mg} \mathrm{L}^{-1}$ \\
\hline Sulfato & $18 \mathrm{mg} \mathrm{L}^{-1}$ \\
\hline Sulfeto total & $0,012 \mathrm{mg} \mathrm{S}^{-}-\mathrm{L}^{-1}$ \\
\hline Surfactantes & $<0,11 \mathrm{mg} \mathrm{L}^{-1}$ \\
\hline Turbidez & $8,58 \mathrm{UNT}^{-1}$ \\
\hline Zinco total & $0,14 \mathrm{mg} \mathrm{L}^{-1}$ \\
\hline
\end{tabular}

O nível de $\mathrm{pH}$ da amostra analisada ficou bem abaixo do valor típico recomendado [13], caracterizando um ambiente ácido. O uso de água de alimentação com pH ácido e alta concentração de cloretos favorece o processo de corrosão de várias maneiras, como a corrosão ácida, seguida pela quebra da camada passiva de aço [14]. Embora a autoclave possuísse um sistema de tratamento de água compacto, a manutenção dos filtros e da membrana de osmose encontrava-se em atraso conforme manual do fabricante, resultando não apenas em uma baixa taxa de fluxo, mas também na redução da eficiência do sistema.

\subsection{Análise de falhas: uso do FMEA e do FTA}

A aplicação da técnica FMEA (Tabela 2) levantou três hipóteses principais: água de alimentação fora de especificação, processo de fabricação incorreto e operação inadequada do gerador de vapor. Com relação ao modo de falha representado pela ruptura da estrutura, pode-se salientar que um correto monitoramento da integridade estrutural pode agilizar o processo de manutenção, uma vez que a ocorrência de vazamentos de água líquida e em vapor e pontos com sinais evidentes de corrosão devem ser reparados de maneira periódica, a fim de evitar o colapso total da estrutura. Além disso, manutenções preventivas devem ser realizadas, pois além de prejuízos financeiros, decorrentes de manutenções corretivas e de paradas não programadas da máquina, o colapso da estrutura pode causar acidentes fatais aos operadores.

Tabela 2: FMEA do gerador de vapor.

\begin{tabular}{|c|c|c|c|}
\hline FUNÇÃO & MODO DE FALHA & EFEITO & CAUSA \\
\hline $\begin{array}{l}\text { Gerar vapor de } \\
\text { baixa pressão } \\
\text { para esterilizar } \\
\text { equipamentos }\end{array}$ & Ruptura & $\begin{array}{l}\text { i. Vapor para processo não especificado; } \\
\text { ii. Vazamento de água líquida e em vapor; } \\
\text { iii. Danos a pessoas e equipamentos próximos } \\
\text { devido ao risco de explosão; } \\
\text { iv. Item inoperante; } \\
\text { v. Esterilização incompleta; } \\
\text { vi. Formação de pontos de oxidação e corrosão } \\
\text { na estrutura de caldeiras; } \\
\text { vii. Vida útil reduzida do sistema. }\end{array}$ & $\begin{array}{l}\text { i. Água de alimentação fora } \\
\text { da especificação; } \\
\text { ii. Processo de fabricação } \\
\text { inadequado; } \\
\text { ii. Operação inadequada. }\end{array}$ \\
\hline
\end{tabular}

Com base nas causas do modo de falha por ruptura da estrutura, percebe-se a necessidade de procedimentos padronizados e atualizados para a operação da máquina, uma vez que a operação inadequada pode levar à uma deterioração prematura da estrutura, além de influenciar negativamente no processo de esterilização, função principal da caldeira em estudo.

Salienta-se também que o objetivo da ferramenta FMEA é aumentar a confiabilidade do processo analisado, além de direcionar de forma mais precisa o uso de recursos para a eliminação de potenciais causas 
de um determinado defeito [7]. Portanto, para a melhoria do processo, torna-se necessário um levantamento sobre as devidas ações a serem realizadas para mitigar as causas encontradas e um posterior controle periódico das medidas implementadas, de modo a garantir a correta manutenção da integridade estrutural.

A ferramenta FTA, por sua vez, ajudou a denotar as relações hierárquicas dos eventos que levam à ruptura da estrutura. Com base no FTA (Figura 5), houve uma compreensão sobre a influência de fatores químicos (água de alimentação com pH baixo e íons cloreto) no processo de corrosão, ressaltando a necessidade de compreender o grau de agressividade desse material à estrutura da máquina e a efetividade dos filtros e da membrana de osmose do sistema. Para isso, a evolução dessas reações químicas e a decorrente corrosão poderiam ser estudadas, de modo a aumentar a confiabilidade da estrutura e compreender a taxa de degradação da mesma.

Erros operacionais no processo de soldagem também foram considerados relevantes para uma possível ruptura da estrutura. Ações como o uso de procedimentos padronizados, com base em normas certificadas, são essenciais para uma boa qualidade na soldagem. Na soldagem de arco elétrico com eletrodo revestido, o uso de parâmetros inadequados, como corrente excessiva e arco muito longo, pode levar à formação de porosidades e, consequentemente, à degradação da junta soldada [15].

Outro fator importante é a realização periódica de treinamentos com os operadores da máquina, ressaltando tanto os procedimentos técnicos necessários para a sua operação quanto os possíveis efeitos de uma ruptura, em termos de riscos à saúde do trabalhador [8].

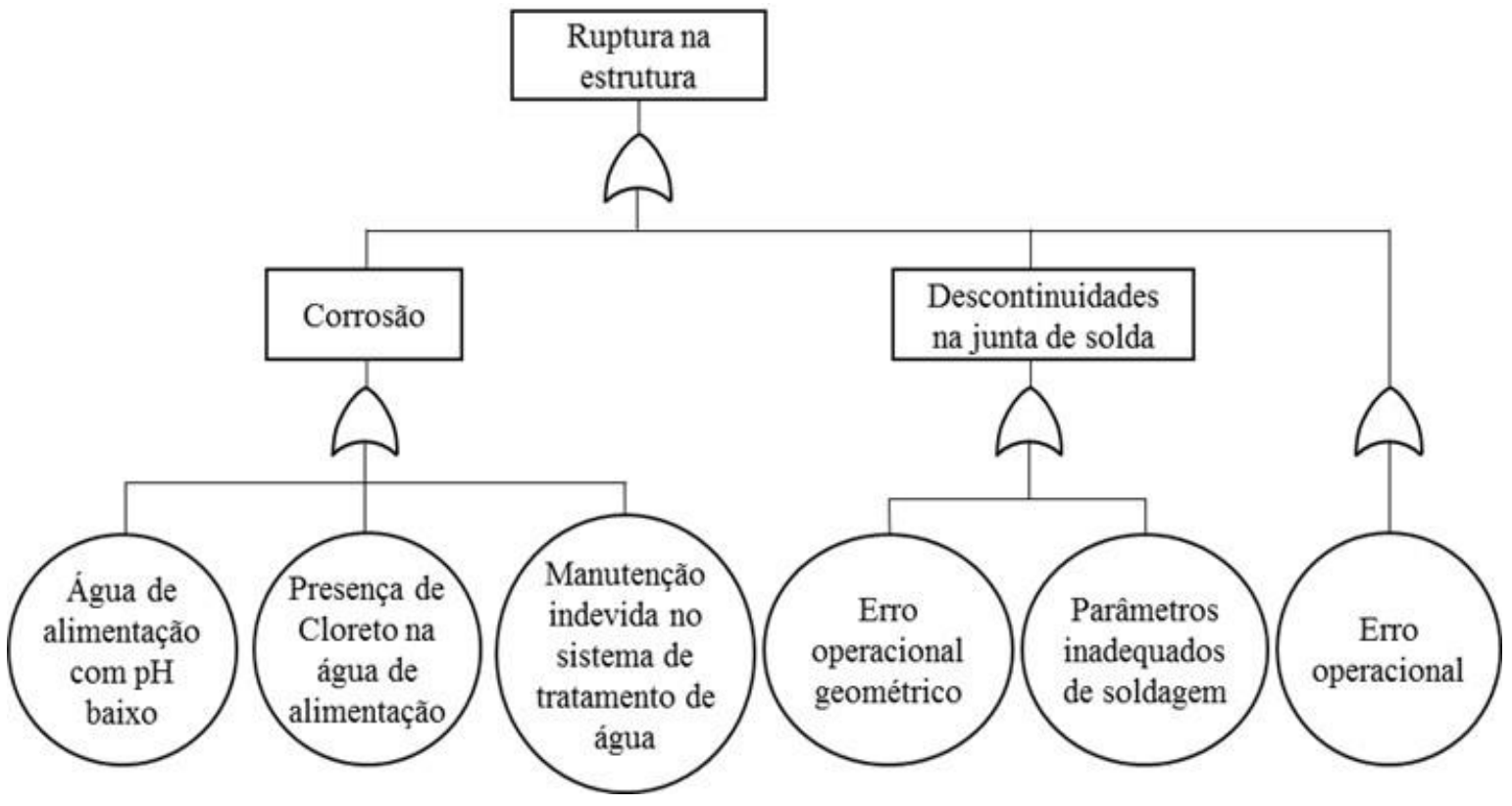

Figura 5: FTA do gerador de vapor.

\section{CONCLUSÕES}

Alterações microestruturais ocorreram pelo processo de soldagem nas regiões da zona fundida, da zona termicamente afetada e do metal de base, assim como falta de penetração do material fundido, desalinhamento de junta e corrosão por pites . A análise físico-química da água, por sua vez, mostrou alta concentração de cloreto e baixo pH da água de alimentação.

Após a aplicação das técnicas FMEA e FTA, foi possível notar que não há uma causa única para a ruptura do gerador de vapor, mas uma soma de fatores associados a baixa qualidade da água de alimentação da caldeira e erros durante o processo de soldagem que atuam como concentradores de tensão. Para controlar o processo corrosivo nos sistemas de geração de vapor e aumentar o seu ciclo de vida, tratamentos externos e internos na água de alimentação devem ser realizados juntamente com a inspeção na autoclave. Para isso, os filtros e as membranas existentes devem ser mantidos no sistema para garantir bons parâmetros de água e uma operação correta do sistema. Por fim, salienta-se a importância de um procedimento operacional padronizado, que auxilie na correta utilização da máquina, garantindo um bom funcionamento e evitando possíveis acidentes, decorrentes da ruptura inesperada da estrutura. 


\section{AGRADECIMENTOS}

Os autores agradecem ao Instituto Federal de Educação, Ciência e Tecnologia do Espírito Santo - IFES e ao Programa de Pós-Graduação em Engenharia Mecânica - PPGMEC da Universidade Federal de Minas Gerais - UFMG pela estrutura física e apoio. Os autores agradecem às agências brasileiras CAPES, CNPq, FINEP e FAPEMIG pelo apoio financeiro.

\section{BIBLIOGRAFIA}

[1] BERTANI, N.G., OLIVEIRA, T.A., ALVERNAZ, S.S., "Qualitative analysis among chemical and physical sterilization methods", Educação, Meio Ambiente e Saúde, v. 3, n. 1, pp. 223-237, 2008.

[2] GIAROLLO, D.F., MAZZAFERO, C.C.P., MAZZAFERO, J.A.E., "Efeito do metal de adição na resistência ao desgaste por deslizamento de um aço estrutural soldado por GMAW", Matéria (Rio J.), v. 24, n. 3, e12464, 2019.

[3] GHALAMBAZ, M., ABDOLLAHI, M., ESLAMI, A., et al., "A case study on failure of AISI 347H stabilized stainless steel pipe in a petrochemical plant", Case Studies In Engineering Failure Analysis, v. 9, n. 1, pp. 52-62, 2017.

[4] PANAHI, H., ESLAMI, A., GOLOZAR, M.A., et al., "Failure analysis of type 304 stainless steel amine exchanger sheets in a gas sweetening plant", Case Studies In Engineering Failure Analysis, v. 9, n. 1, pp. 8798, 2017.

[5] SANTOS JR., A.G.; BIEHL, L.V.; ANTONINI, L.M. "Effect of chemical passivation treatment on pitting corrosion resistance of AISI 430 and AISI 316L stainless steels", Matéria (Rio J.), v. 23, n. 1, e$11957,2018$.

[6] GONÇALVES, R.B.; TERRONES, L.A.H.; ROCHA, R.P. "Effect of Solubilization and Stabilization Heat Treatment on Microstructure of a 347 Stainless Steel Welded Joint”, Matéria (Rio J.), v. 22, n. 1, e11784, 2017.

[7] JOO, B., KIM, S., KIM, S., et al., "FMEA for the reability of hydroformed flanged part for automotive application", Journal of Mechanical Science and Technology, v. 27, n. 1, pp. 63-67, 2013.

[8] LEU, S.-S., CHANG, C.-M., "Bayesian-network-based safety risk assessment for construction projects", Accidents Analysis and Prevention, v. 54, pp. 122-133, 2013.

[9] MIRANDA, B.O.A.P., ZANCANELLA, A.C.B., MAZIERO, R., et al., "Análise de danos e falhas em um gerador de vapor", Acta Mechanica et Mobilitatem, v. 4, n. 1, pp. 1-4, 2019.

[10] WANG, Y., WU, X., LI, X., et al., "Combined effects of prior plastic deformation and sensitization on hydrogen embrittlement of 304 austenitic stainless steel", International Journal of Hydrogen Energy, v. 44, n. 13, pp. 7014-7031, 2019.

[11] BARLA, N.A., GHOSH, P.K., "Simulated Stress-Induced Sensitization Study for the Heat-Affected Zone of the 304LN Stainless Steel Weld Using a Thermomechanical Simulator", Metallurgical and Materials Transactions A, v. 50, n. 3, pp. 1283-1293, 2019.

[12] DI, X., TONG, M., LI, C., et al., "Microstructural evolution and its influence on toughness in simulated inter-critical heat affected zone of large thickness bainitic steel", Materials Science and Engineering: A, v. 743, n. 1, pp. 67-76, 2019.

[13] AGÊNCIA NACIONAL DE ÁGUAS. "Manual de conservação e reuso de água na agroindústria sucroenergética". Brasília: ANA, 288 pp. 2009.

[14] FUNDÃO, A.S., SILVA, F.Z., GARCIA, D.P., et al., "Failure by corrosion in a sea water overboard line", Univap, v. 22, n. 41, pp. 5-12, 2016.

[15] RAJKUMAR, R., VEDHI, C., "A study of corrosion protection efficiency of silica nanoparticles acrylic coated on mild steel electrode", Vacuum, v. 161, n. 1, pp. 1-4, 2019.

\section{ORCID}

Beatriz Ottoni Azevedo Porto Miranda

Antonio Carlos Barbosa Zancanella

Rômulo Maziero

Bruno Dorneles de Castro

Juan Carlos Campos Rubio https://orcid.org/0000-0001-6882-2092

https://orcid.org/0000-0002-0919-3852

https://orcid.org/0000-0002-5739-1941

https://orcid.org/0000-0003-2895-9697

http://orcid.org/0000-0001-6187-6442 